\title{
A preliminary study on clinical problems in acupuncture treatment for cancer pains.
}

\author{
Jia-Bin Tai ${ }^{1,2}$, Liang Hong ${ }^{1}$, Min Fu ${ }^{1}$, Jia Xu${ }^{1}$, Shui-Jun Gu ${ }^{1 *}$, Jian-Qiao Fang ${ }^{2 *}$ \\ ${ }^{1}$ Zhejiang Chinese Medicine University, Zhejiang, Hangzhou, 310053, PR China \\ ${ }^{2}$ Affiliated Xiaoshan First Hospital of Hangzhou Normal University Medical School, PR China
}

\begin{abstract}
In this study, recent literatures and clinical research experience were collected and analyzed. The main problems of acupuncture in the treatment of cancer pains were summarized and listed as follows. (1) Cancer pains have complex causes and diverse symptoms, and some mixed pains need other drugs for adjuvant treatment. (2) Limited degree of pain control. For mild to moderate pains (pains with a numerical rating scale [NRS] score of $<4$ points), good results can be achieved; while for severe pains (pains with an NRS score of $\geq 4$ points), drug treatment must be concurrently performed. (3) Analgesia timeliness. After treatment, the majority of patients felt that their pains gradually aggravated. Hence, these patients were returned to pre-treatment level at 4-6 $\mathrm{h}$ after treatment. (4) Diverse selection of acupoints. In China, most researchers use the TEAS method, where they select acupoints based on syndrome differentiation; and researchers sometimes use local acupoint selection as adjuvant means. Most foreign researchers treat cancer pain using the TENS method. (5) It is difficult to conduct randomized double-blind controlled studies of this treatment. Because, the treatment of acupuncture is easier to be identified by patients with acupuncture or electro-acupuncture experience.
\end{abstract}

Keywords: Acupuncture treatment, Cancer pains, TEAS method.

Accepted on November 24, 2017

\section{Introduction}

With the increase in the number of cancer patients, cancer pain has become one of the important factors that affect the quality of life of cancer patients, and a serious global public health problem [1]. The World Health Organization (WHO) threestep analgesic ladder is the main drug analgesia method used for the treatment of cancer pain. Morphine, oxycodone, fentanyl and other opioid drugs have become the first choice for moderate-severe cancer pain. The proportion of pain control can reach $90 \%[2,3]$. The long-term use of opioid drugs induces many problems, of addiction, resistance, and gastrointestinal and nervous systemic adverse reactions [4,5], and the cause of pains is complex. Furthermore, the single use of opioids has poor effects on neuropathic pain. Therefore, acupuncture analgesia treatment, a traditional Chinese medicine method, has become an alternative therapy that has been extensively applied in clinical practice in recent years; and this treatment method has advantages of safety, quick effect and the absence of adverse drug reactions [6-8]. In particular, when concurrently used with opioids, it has the effects of improving analgesia, reducing toxicity, enhancing efficacy and improving the quality of life of patients $[9,10]$.

At present, published clinical literatures involve a variety of diseases, in which the locations and nature of pain were different, a strict randomized control was not performed, and the double-blinded principle was not adhered to. This lead to inconsistent research results, and the effectiveness of acupuncture in the treatment of cancer pain was challenged [11-13]. As treasures of the traditional Chinese medicine (TCM), acupuncture and Chinese herbal medicine have a history of more than 2,500 years and have achieved sound effects in the clinical practice [14]. The effects of acupuncture has usually been demonstrated by biological regulations of physiological and pathological processes [15], which are inherent responses of human beings and of great importance in the life science research [16]. On the basis of clinical experience, the main problems that may affect the efficacy and results of the study were summarized, in order to provide basis for the standardization of subsequent studies.

\section{The Causes of Cancer Pain are Complex, Symptoms are Diverse, and Some Mixed Pains Require other Auxiliary Drugs}

Cancer pain is pain caused by cancers or cancer-related factors. Its main causes include the local infiltration or invasion of tumors, the compression of peripheral tissues, nerves and blood vessels, or bone, pleura, liver, skin and brain metastasis, or postoperative nerve injury and psychological factors [17-19]. 
Furthermore, cancer pain is a chronic pain that has a unique and complex mechanism. In animal models, pain-related behaviors such as hyperalgesia and hypersensitivity have been observed, and unique neurochemical changes occur in corresponding segments of the spinal cord. The occurrence of cancer pain is associated with peripheral and central afferent nerve sensitizations. In the early stage of cancer pain, the main causes are the primary afferent nerve sensitization induced by tumor cells, pain-inducing substances produced by inflammatory cells, and the persistent activation of osteoclasts. In the late stage of cancer pain, the compression and injury of nerve induced by tumor growth are involved in the occurrence of cancer pain [20]. Acupuncture or electro-acupuncture serves as an auxiliary means to relieve pain, and the main mechanism is to produce endorphin and dynorphin through local electrical stimulation on the body [21-24]. This increases the body's pain threshold, inhibits the stimulation of pain to afferent pathways, and regulates local pain-induced factors; thereby relieving pain [25-27].

In clinical applications, electro-acupuncture stimulation has a satisfactory effect on pure chronic cancer pains in local muscle tissues such as sores, distending pain and stings. For visceral pain caused by the space-occupying lesions of the liver, lung and pancreas, since the pain site is deep, the local selection of acupoints has poor effects. Hence, the treatment acupoints should also be selected based on syndrome differentiation, and drug therapy is often required. For patients with neuropathological pain induced by bone metastasis or nerve invasion, since some patients have characteristics of burning sensation in the limbs, numbness, and even hyperalgesia causing pain when the body is touched, electro-acupuncture therapy should be combined with non-steroidal drugs, antiepileptic drugs, anti-anxiety drugs and other adjuvant therapies $[9,28]$. In addition, bone metastatic cancer pain with the invasion of vertebral bodies, ribs and other sites, can be induced by activity or cough. Hence, repeated electroacupuncture stimulation (4-6 times per day or more) is required to control the pain.

\section{Limited Degrees of Pain Control}

When electro-acupuncture stimulation is given to treat cancer pain, the best analgesic effect can be achieved by adjusting the frequency, intensity and waveform of the acupuncture [29]. Within a certain range, the current strength is positively correlated with the analgesic effect [30]. However, it is not entirely true that the stronger the current strength is, the better the analgesic effect becomes. In the process of treatment, if the current strength reaches a certain degree where patients experience numbness and a tingling sensation, and this current strength is called the "sensation threshold". If this current strength slightly increases, the patient will develop a sting sensation. The current strength can cause a pain sensation called, the "pain threshold" of the current. The sensation threshold and pain threshold varies from person to person, and the differences are also great under various pathological conditions. In general, the current strength between the sensation threshold and pain threshold is the most suitable strength for treatment [29].

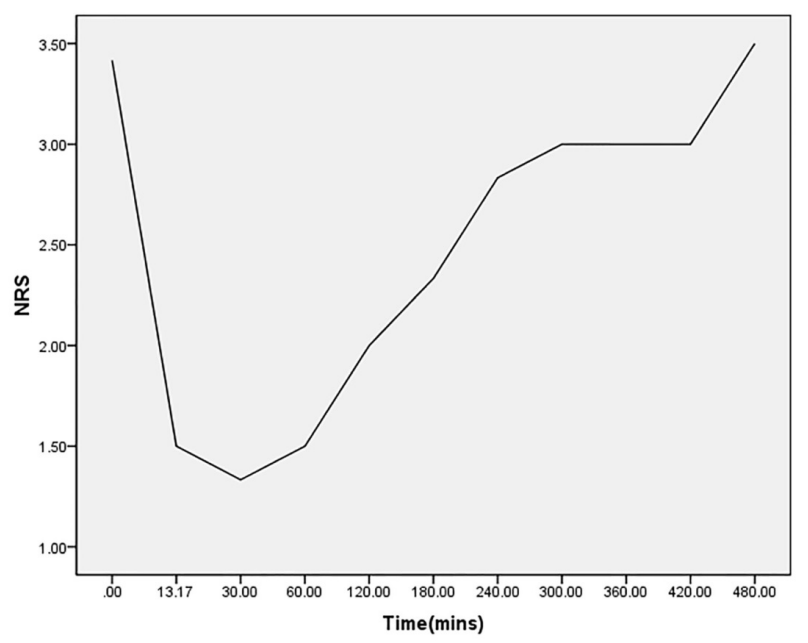

Figure 1. The comparison of NRS score after the first treatment; Note: 2 curves in figure presents downtrend, which were related with participation of the drugs during the research.

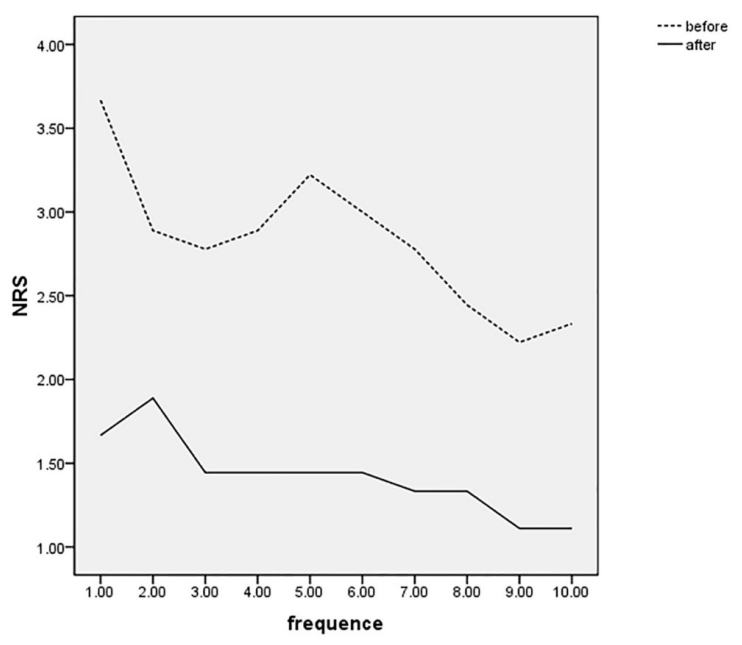

Figure 2. The comparison of NRS score before and after a course of treatment; Note: 2 curves in figure presents downtrend, which were related with participation of the drugs during the research.

With the example of Korean electro-acupuncture apparatus $200 \mathrm{~A}$, the dilatational wave is often used for analgesia, and the magnitude of the current differs to a certain extent $h$, according to the site and distance of the selected acupuncture point. The tolerated strength between both sides of the spine in the back is the maximum, which is up to $30-50 \mathrm{~mA}$; and in the limbs, chest and abdomen, it is often maintained at 8-20 mA. Current strength exceeding the pain threshold may cause severe muscle spasm, inducing needle breaking, needle curving and fainting during the acupuncture treatment. This may even cause burns, fractures and ventricular fibrillation that would endanger the life of the patient.

In the process of the electro-acupuncture analgesia treatment, in addition to the parameter limitations of the current, the 
extent of pain control through the suitable current parameter is also limited. The author has stimulated on Jiaji (EX-B2), Neiguan (pc 6), Hegu (L14), zusanli (st 36), sanyinjiao (sp 6) in patients with bone metastatic pain in vertebral bodies. The study revealed that for severe pain (NRS score $\geq 7$ points), the curative effect was poor and opioid drugs were necessarily used. For mild to moderate pain (NRS score $\leq 6$ points), treatment with transcutaneous electrical acupoint stimulation (TEAS) alone or TEAS combined with drugs can be used according to patient tolerance (Figures 1 and 2). As shown in Figure 1, the average difference in NRS score before and after electro-acupuncture analgesia was 1.92, and the extent of decrease in pain score was 1-3 points. In Figure 2, a comparison of NRS scores after a course of treatment is shown (two times per day, for five days). The curative effect range of electro-acupuncture analgesia was within 1.2-2.0, and the average was 1.4. These results reveal that when comparing either a single or multiple treatments, the analgesia range of electro-acupuncture for treating cancer pain is limited. For mild to moderate pain (NRS score $<4$ points), the application of electro-acupuncture alone can achieve good results. However, for moderate-severe pain (NRS score $\geq 4$ points), in order to obtain a better analgesic effect, drug therapy must be combined.

\section{Analgesia Timeliness}

For either TENS or TEAS, or acupuncture plus electroacupuncture analgesia, in addition to the limited degree of pain control, the analgesic effect has timeliness [31]. Previous studies have revealed that within approximately $13.17 \mathrm{~min}$ (4-30 $\mathrm{min}$ ) after electro-acupuncture stimulation, pain relief was achieved and pain score decreased by 1-3 points. Furthermore, the best analgesic effect was achieved after 30 min of treatment. After the end of treatment, the majority of patients felt that their pains gradually aggravated with time. Hence, these patients were return to pre-treatment levels at 4-6 $\mathrm{h}$ after the treatment (Figure 1). Even though patients had been repeatedly treated with electro-acupuncture for analgesia, pretreatment pain score was still higher (NRS score was 2.82 points) and significantly different from post-treatment levels. This further suggests that the analgesic effect has timeliness (Figure 2). In a study, the method of simple increase in the frequency of acupuncture (two times or even 5-6 times per day) was used as an anti-pain treatment for patients with mild to moderate cancer pain; and the majority of patients turned to or additionally received opioid drugs (sustained-release formulation) to control pain caused by repeated pains, sleep interruption, etc. Results revealed that for patients with chronic and persistent moderate to severe pains (NRS score $\geq 4$ points), increasing the frequency of treatment does not solve the problem of timeliness in electro-acupuncture stimulation.

\section{Diverse Selection of Acupoints}

In China, most researchers use acupuncture and TEAS to treat cancer pain. They select acupoints mainly based on syndrome differentiation, and sometimes use local acupoint selection as adjuvant means [32]. During the treatment, the location of the cancer pain needs to be distinguished, and the meridian where the pains belong to should be determined. Furthermore, acupoints with its corresponding main therapeutic effect or distal acupoints along the meridian are selected for treatment. Details are described as follows.

In this study, the acupoint selection treatment based on syndrome differentiation [33,34] mainly includes the following: (1) The xi-cleft points serve as the dominant acupoints. The xi-cleft points are the deep gathering places of the qi from various meridians, and are mainly used for the treatment of the acute pain of cancer in internal organs. For example, kongzui is used for lung cancer, $\operatorname{lr} 6$ is used for liver cancer, li 7 is used for colorectal cancer, yanglao is used for small intestine cancer, liangqiu is used for gastric cancer, and Jinmen is used for bladder cancer and prostate cancer. (2) Endto-end acupoint selection: End-to-end acupoint selection is a method where the initial acupoint and terminal acupoint in the meridians are stimulated to treat the symptom, in which cancer pains radiate in the viscera to the meridians in the limbs. For example, lung cancer-induced chest pain with radiation from the supraclavicular fossa to the forearm belongs to lung meridian diseases. Hence, zhongfu and shaoshang acupoints should be selected. (3) Shu-Mu acupoints: Back-Shu acupoints are Acupoints in the back waist where the viscera qi is infused, while front-Mu acupoints are Acupoints in the chest and abdomen where the viscera qi acummulates. Shu-Mu acupoints are mainly used for weak patients with long-term cancer pain in the viscera. For example, lung cancer is treated at feishu and zhongfu, liver cancer is treated at ganshu and qimen, colorectal cancer is treated at dachangshu and tianshu, bladder cancer is treated at pangguangshu and zhongji, small intestine cancer is treated at bl 27 and guanyuan, gastric cancer is treated at bl 21 and zhongwan, and so on. (4) Lower confluent points for bowel diseases: According to the "lower confluent points for bowel diseases" theory in the article, "Lingshu. Performance of bowel diseases induced by pathogens", cancer pain in the bowel can be treated at the lower confluent points. For example, gastric cancer is treated at zusanli, colorectal cancer is treated at shangjuxu, small bowel cancer is treated at xiajuxu, bladder cancer is treated at weizhong, and gallbladder cancer is treated at yanglingquan. (5) Eight confluence points: The eight confluence points refer to acupoints where the essence from the viscera, $\mathrm{Fu}$, qi, blood, muscle, meridian, bone and bone marrow converges. According to the means of regulating cancer pain, the corresponding confluence point is selected. For example, viscera cancers are treated at zhangmen, $\mathrm{Fu}$ cancers are treated at zhongwan, and bone cancers are treated at dazhu. (6) Attention to regulating mental activities and soothing of the liver: The "Huangdi's Classic on Medicine" and commentators over the ages elucidate the relationship between pain and the mind from many aspects. For example, the "Plain Questions-Essential and Important Major Theories" states: itching, pain and ulcer are regulated by the heart meridian. After diagnosis, fear of pain and other states of mind play important roles in the lives of cancer patients, disease progression and physical deterioration; and loss of 
confidence in the treatment would further aggravate the pain. Therefore, soothing acupoints of neiguan, tailing and xinshu are often stimulated to treat cancer pain. In addition, more than $25 \%$ of cancer patients are accompanied by different degrees of depressive symptoms. Hence, the "four gate points" and the qimen point, which have the function to soothe the liver, can be stimulated [35].

Local acupoint selection mainly refers to acupuncture at the pain points. The needle is exerted at different positions, according to the different parts of cancer pains. Generally, 3-5 points with obvious tenderness around the pain site are selected for treatment [9]. According to currently published literatures, most cases involve dialectical therapy. Hence, a standard for acupoint selection could not be unified, and the same disease may be treated at different acupoints. Effective evidence of clinical research is limited to single-blind randomized controlled trials or personal experience. This method has poor repeatability, and is difficult to popularize. Most of the foreign researchers have treat cancer pain using the TENS method [36-38], where the local area of pain is stimulated for treatment, positioning is simple, and the procedures are easily mastered. The method of selecting points has greater limitations on the treatment of patients with multiple sites of pains. In addition, this method lacks the overall concept and participation of the meridian theory. Therefore, it does not achieve the best clinical efficacy. Furthermore, it is easy to identify patients with acupuncture or electro-acupuncture experience through the presence of needling sensations. Therefore, the randomized double-blinded controlled study of acupuncture or electro-acupuncture in the treatment of cancer pain is difficult to carry out. Hence, clinical studies are often single-blinded, taking high or low frequency, or strong or weak current, as controls.

\section{Conclusion}

Due to advantages high safety, high efficiency, fewer side effects and no addiction, acupuncture has become an indispensable clinical treatment [39]. In the treatment of cancer pain, electro-acupuncture and other non-drug alternative therapies would become important auxiliary methods. Electroacupuncture or acupuncture treatment for cancer pain still has many shortcomings, which limit its clinical promotion and application. Recommendations: (1) In studies, patients with a single disease, single pain location and nature should be selected as research subjects, and local acupoint selection combined with dialectical acupoint selection should be adopted, in order to achieve a better analgesic effect. (2) Researchers can carry out controlled studies between different methods. For example, researchers can conduct studies that compare the curative effect of the TENS and TEAS methods, in order to clarify the relationship between different acupoints and curative effects. (3) Moderate-severe cancer pain can be treated with drug therapy, and electro-acupuncture or acupuncture alone is not recommended. (4) Small or buttontype press-triggering micro electro-acupuncture devices should be developed, which would be more practical and could be concurrently used with the analgesic pump. This study was written based on the clinical experience of the author, and intends to offer a few commonplace remarks by way of introduction, allowing others scholars to come up with valuable opinions. The author aspire the cooperation between China and the West, in order to seek a breakthrough in technology that would benefit patients with cancer pains.

\section{Acknowledgement}

Fund Project: Hangzhou science and technology plan health project (NO. 20150633).

\section{References}

1. Regaard A. The principles of pain management in advanced cancer. Br J Community Nurs 2000; 5: 382-386, 388.

2. Song GP, Jiang YQ, Gou LQ, Li XF. Clinical observation on the treatment of cancer pain by morphine hydrochlorid sustained release tablets. J Basic Clin Oncol 2006; 19: 229-230.

3. Mercadante S, Ferrera P, Villari P, Casuccio A. Opioid escalation in patients with cancer pain: the effect of age. J Pain Symptom Manage 2006; 32: 413-419.

4. Li Q, Luo J. The research progress of treating chronic moderate and severe pain by opioid medicines. Chinese J Pain Med 2011; 17: 116-119.

5. Wang JJ. A survey of cancer pain status in Shanghai. Oncology 2008; 74 Suppl 1: 13-18.

6. Loh J, Gulati A. The use of transcutaneous electrical nerve stimulation (TENS) in a major cancer center for the treatment of severe cancer-related pain and associated disability. Pain Med 2015; 16: 1204-1210.

7. Garcia MK, Driver L, Haddad R, Lee R, Palmer JL, Wei Q, Frenkel M, Cohen L. Acupuncture for treatment of uncontrolled pain in cancer patients: a pragmatic pilot study. Integr Cancer Ther 2014; 13: 133-140.

8. Chiu HY, Hsieh YJ, Tsai PS. Systematic review and metaanalysis of acupuncture to reduce cancer-related pain. Eur J Cancer Care (Engl) 2017; 26.

9. Hou GY, Zhang LX, Lu LJ, Li X. The clinical discussion of acupuncture combined with drugs to relieve cancer pain in elderly. Chinese J Trauma Disability Med 2015; 23: 79-80.

10. Zeng Y, Luo T, Finnegan-John J, Cheng AS. Meta-analysis of randomized controlled trials of acupuncture for cancerrelated fatigue. Integr Cancer Ther 2014; 13: 193-200.

11. Adam H, Michael BI, Karen RA, Mark JI, Karen SH, Stephen OG. Transcutaneous Electric Nerve Stimulation (TENS) for cancer pain in adults. Cochrane Database Syst Rev 2012; 14: CD006276.

12. Karen R, Stephen OG, Michael BI, Mark JI, Karen SH, Robert SD. A cochrane systematic review of transcutaneous electrical nerve stimulation for cancer pain. J Pain Symptom Manage 2009; 37: 746-753.

13. Choi TY, Lee MS, Kim TH, Zaslawski C, Ernst E. Acupuncture for the treatment of cancer pain: a systematic 
review of randomised clinical trials. Support Care Cancer 2012; 20: 1147-1158.

14. Cheung F. TCM: Made in China. Nature 2011; 480: S82-83.

15. Zhao ZQ. Neural mechanism underlying acupuncture analgesia. Prog Neurobiol 2008; 85: 355-375.

16. Yang YQ, Yan C, Branford-White CJ, Xiang-Yu H. Biological values of acupuncture and chinese herbal medicine: impact on the life science. Evid Based Complement Alternat Med 2014; 2014: 593921.

17. Wu GQ, Sun Y, Luo J, Yang P, Xu JY, Li PW, Wu JD, Wang X, Shi B, Chen J, Lv X, Zhang ZQ, Zhang YL, Li YS, Gao YW, Ma BR, Zhang HP. A survey of pain and quality of life (QOL) of cancer patients in China. Chinese J Pain Med 1995; 1: 66-75.

18. Liu RK, Wang J, Ji XQ, Liu ZM. Epidemiological survey on the prevalence of cancer related pain. Chin J Drug Depend 2005; 14: 354-359.

19. Li QS. The mechanism and treatment principle of cancer pain. Tumor 1997; 17:183-185.

20. Wang MM, Wang JJ. The update study of the initiation mechanism and drug therapies of cancer pain. Chinese Clin Oncol 2011; 16: 662-666.

21. Fei H, Xie GX, Han JS. Low and high frequency electroacupuncture stimulation release met-enkephalin and dynorphin A in rat spinal cord. Chinese Sci Bull 1987; 32: 1496-1501.

22. He CM, Han JS. Attenuation of low rather than high frequency electroacupuncture analgesia following microinjection of $\beta$-endorphin antiserum into the periaqueductal gray in rats. Acupunct Sci Int J 1990; 1: 19-27.

23. Chen XH, Guo SF, Chang CG, Han JS. Optimal conditions for eliciting maximal electroacupuncture analgesia with dense-and-disperse mode stimulation. Am J Acupunct 1994; 22: 47-53.

24. Huang C, Wang Y, Chang JK, Han JS. Endomorphin and $\mu$ opioid receptors in mouse brain mediate the analgesic effect induced by $2 \mathrm{HZ}$ but not $100 \mathrm{HZ}$ electroacupuncture stimulation. Neurosci Lett 2000; 294: 159-162.

25. Zhao WL, Zhao WS, Huang HW, Zhong LL. Effects of electroacupuncture on the hypothalamic $\beta$-endorphin in rats with bone cancer pain. JCAM 2013; 29: 69-72.

26. Sima L, Liu BT, Li JC, Liu NG, Zhang Y. Effect of electroacupuncture treatment on pain behavior and calcitonin-gene related peptide expression in cancer induced bone pain-morphine tolerance rat model. Chin J Behac Med Brain Sci 2013; 22: 388-390.

27. Chen YT, Du JY, Liang Y, Wu SF, Zhang D, Fang JQ. Analgesic effect of electroacupuncture on spinal PDYN and POMC mRNA expression in rats with subcutaneous tumorinduced pain. J Zhengjiang Chinese Med University 2015; 3: 221-226.
28. Mao YQL, Yen DH, Mi WY, Liu Q, Wang YQ. Analgesic effects of electroacupuncture combined with Celebrex on rats with tibial cancer pain. J Chinese Integrat Med 2008; 6: 830-835.

29. Zhuang YY, Huang XQ, Chen X, Ye XR. A review on application of electrical stimulation parameters. CJCM 2015; 23: 139-141.

30. Kuai L, Chen H, Zhang TT, Yang YH. Study on dose-effect relationship of electroacupuncture with different current intensities alleviating tibial cancer pain and inhibition of expression of spinal GFAP in rats. Chinese Acupuncture Moxibustion 2012; 4: 331-337.

31. Guo RX, Zhang LY, Gong YM, Zhang BG. Chinese medicine soup meridian rehabilitation apparatus in treatment of tumor bone metastases pain 286 cases. Med J Lanzhou Command PLA 1993; 2: 24-24.

32. Huang J, Ding JY. Analysis of traditional Chinese medicine treatment of cancer pain. China Higher Med Edu 2013; 9: 134-135.

33. Zheng K, Song J, Gao Y, Dong BQ. Clinical observation of acupuncture relieving moderate and sever cancer pain. J Liaoning University TCM 2015; 17: 19-21.

34. Chen LX, Xie Q, Wang YQ. Effect of electric acupuncture treatment of cancerous neuralgia. J Cervicodynia Lumbodynia 2014; 35: 76-77.

35. Fu WB, Zhang B. The thought of acupuncture treatment for cancer pain. Shanghai J Acu-mox 1999; 4: 32-33.

36. Bergeron-Vézina K, Corriveau H, Martel M , Harvey MP, Léonard G. High-and low-frequency transcutaneous electrical nerve stimulation does not reduce experimental pain in elderly individuals. Pain 2015; 156: 2093-2099.

37. Loh J, Gulati A. The use of transcutaneous electrical nerve stimulation (TENS) in a major cancer center for the treatment of severe cancer-related pain and associated disability. Pain Med 2015; 16: 1204-1210.

38. Loh J, Gulati A. Transcutaneous electrical nerve stimulation for treatment of sarcoma cancer pain. Pain Manag 2013; 3: 189-199.

39. Lu Y, Tian WW, Li KP. The research progress of acupuncture in the treatment of pain. JETCM 2016; 10: 1915-1917.

\section{*Correspondence to}

Jian-Qiao Fang

Zhejiang Chinese Medicine University

PR China

Shui-Jun $\mathrm{Gu}$

Affiliated Xiaoshan First Hospital of Hangzhou Normal University Medical School

PR China 